IFT-P.026/98

\title{
Constraints on Electroweak Contact Interactions from LEP and Tevatron Data.
}

\author{
Alexander Belyaev ${ }^{1,2}$ and Rogerio Rosenfeld ${ }^{1}$ \\ ${ }^{1}$ Instituto de Física Teórica, Universidade Estadual Paulista, \\ Rua Pamplona 145, 01405-900 - São Paulo, S.P., Brasil \\ ${ }^{2}$ Skobeltsin Institute for Nuclear Physics, Moscow State University, \\ 119 899, Moscow, Russian Federation
}

\begin{abstract}
A complete set of dimension-6 effective contact interactions involving Higgs, gauge bosons and quarks is studied. Limits on the coefficients of these new operators are obtained from the experimental values of the $Z$ and $W$ gauge bosons widths.
\end{abstract}

\section{INTRODUCTION}

There have been extensive theoretical and phenomenological studies of physics beyond the Standard Model (SM) in recent years. This new physics could be revealed, for example, in studies of anomalous couplings that are either different or absent at tree level in the SM. Studies for such couplings include searches for anomalous gauge boson self-couplings, Higgs boson couplings and top quark couplings.

The most general phenomenological parametrization for new phenomena beyond the SM can be achieved by means of an effective Lagrangian [1] that involves operators with dimension higher than four, containing the relevant fields at low energies and respecting 
the symmetries of the Standard Model. The effective Lagrangian approach is a modelindependent way to describe new physics that can occur at an energy scale $\Lambda$ much larger than the scale where the experiments are performed. New particles present at the energy scale $\Lambda$ are integrated out at low energies, generating higher dimensional operators. The effective Lagrangian depends on the particle content at low energies and here we assume that the Higgs boson can be light, being present in the higher dimensional operators, in addition to the electroweak gauge bosons and fermions, and the SM symmetries are linearly realized [2,3]. In what follows we adopt the notation of Buchmüller and Wyler in ref. [2].

Limits on operators modifying the electroweak and Higgs bosons interactions but leaving the fermion interactions untouched have been obtained from studying many different processes 411$]$. In this short note we concentrate on possible contact interactions involving Higgs, gauge bosons and quarks. These interactions are interesting since they could in principle contribute to associated Higgs and gauge boson production at hadron machines and to anomalous top quark couplings. In fact, some of the analogous leptonic contact operators have been used in the study of anomalous Higgs production at LEP2, like $\mathcal{O}_{\Phi l}^{(1)}, \mathcal{O}_{\Phi l}^{(3)}$ and

$\mathcal{O}_{\Phi e}$ 9]. Also the operators like $\mathcal{O}_{\Phi q}^{(1)}, \mathcal{O}_{\Phi q}^{(3)}, \mathcal{O}_{D u}, \mathcal{O}_{D d}, \mathcal{O}_{u W}$ and $\mathcal{O}_{d W}$ have been studied in the context of anomalous top quark production at the Tevatron [12] and $e-\gamma$ colliders [13].

These operators are constrained at tree-level by the decay widths of the $Z$ and $W$ boson. In this paper we obtain limits for each coefficient of a complete set of contact interactions involving Higgs, gauge bosons and quarks, assuming that different generations can have different anomalous couplings. We also write down the specific linear combination of coefficients that are bounded in each process and notice that accidental cancellations could avoid these direct bounds.

\section{EFFECTIVE LAGRANGIAN AND CONTACT INTERACTIONS}

The most general effective Lagrangian containing dimension-6 terms describing contact interactions among electroweak gauge fields, i.e. $\gamma, W^{ \pm}, Z^{0}$, Higgs boson $H$, and quark 
fields is given by [2]:

$$
\begin{aligned}
\mathcal{L}_{\text {eff }}= & \mathcal{L}_{\mathrm{SM}}+\frac{1}{\Lambda^{2}}\left(f_{\Phi q}^{(1)} \mathcal{O}_{\Phi q}^{(1)}+f_{\Phi q}^{(3)} \mathcal{O}_{\Phi q}^{(3)}+f_{\Phi u} \mathcal{O}_{\Phi u}+\right. \\
& f_{\Phi d} \mathcal{O}_{\Phi d}+f_{\Phi \Phi} \mathcal{O}_{\Phi \Phi}+f_{D u} \mathcal{O}_{D u}+f_{\bar{D} u} \mathcal{O}_{\bar{D} u}+f_{D d} \mathcal{O}_{D d}+f_{\bar{D} d} \mathcal{O}_{\bar{D} d}+ \\
& \left.f_{u W} \mathcal{O}_{u W}+f_{u B} \mathcal{O}_{u B}+f_{d W} \mathcal{O}_{d W}+f_{d B} \mathcal{O}_{d B}\right)+ \text { h.c. }
\end{aligned}
$$

with each operator $\mathcal{O}_{i}$ defined as,

$$
\begin{aligned}
\mathcal{O}_{\Phi q}^{(1)} & =i\left(\Phi^{\dagger} D_{\mu} \Phi\right)\left(\bar{q} \gamma^{\mu} q\right) \\
\mathcal{O}_{\Phi q}^{(3)} & =i\left(\Phi^{\dagger} D_{\mu} \tau^{I} \Phi\right)\left(\bar{q} \gamma^{\mu} \tau^{I} q\right) \\
\mathcal{O}_{\Phi u} & =i\left(\Phi^{\dagger} D_{\mu} \Phi\right)\left(\bar{u} \gamma^{\mu} u\right) \\
\mathcal{O}_{\Phi d} & =i\left(\Phi^{\dagger} D_{\mu} \Phi\right)\left(\bar{d} \gamma^{\mu} d\right) \\
\mathcal{O}_{\Phi \Phi} & =i\left(\Phi^{\dagger} \varepsilon D_{\mu} \Phi\right)\left(\bar{u} \gamma^{\mu} d\right) \\
\mathcal{O}_{D u} & =\left(\bar{q} D_{\mu} u\right)\left(D^{\mu} \tilde{\Phi}\right) \\
\mathcal{O}_{\bar{D} u} & =\left(D_{\mu} \bar{q} u\right)\left(D^{\mu} \tilde{\Phi}\right) \\
\mathcal{O}_{D d} & =\left(\bar{q} D_{\mu} d\right)\left(D^{\mu} \Phi\right) \\
\mathcal{O}_{\bar{D} d} & =\left(D_{\mu} \bar{q} d\right)\left(D^{\mu} \Phi\right) \\
\mathcal{O}_{u W} & =\left(\bar{q} \sigma^{\mu \nu} \tau^{I} u\right) \Phi W_{\mu \nu}^{I} \\
\mathcal{O}_{d W} & =\left(\bar{q} \sigma^{\mu \nu} \tau^{I} d\right) \Phi W_{\mu \nu}^{I} \\
\mathcal{O}_{u B} & =\left(\bar{q} \sigma^{\mu \nu} u\right) \Phi B_{\mu \nu} \\
\mathcal{O}_{d B} & =\left(\bar{q} \sigma^{\mu \nu} d\right) \Phi B_{\mu \nu}
\end{aligned}
$$

where $\Phi$ is the Higgs field doublet, which in the unitary gauge assumes the form,

$$
\Phi=\left(\begin{array}{c}
0 \\
(v+H) / \sqrt{2}
\end{array}\right)
$$

with $B_{\mu \nu}$ and $W_{\mu \nu}^{I}$ being the field strength tensors of the $U(1)$ and $S U(2)$ gauge fields respectively. 
We incorporated all the new interactions arising from the effective lagrangian in the Comphep package [14] by using the code Lanhep [15].

For the $Z$ boson, we obtain the modified $Z b \bar{b}$ and $Z c \bar{c}$ vertices which can be written as follows:

$$
\begin{aligned}
\Gamma_{\mu}^{Z b \bar{b}}= & i \frac{e}{2 s_{W} c_{W}}\left[\gamma^{\mu}\left(g_{V}^{d}+g_{A}^{d} \gamma_{5}\right)+\gamma^{\mu}\left(\kappa_{1}^{d V}-\kappa_{1}^{d A} \gamma_{5}\right) / 2+\left(p_{1}^{\mu}-p_{2}^{\mu}\right) \kappa_{2}^{d V} / M_{Z}+\right. \\
& \left.\left(p_{1}^{\mu}+p_{2}^{\mu}\right) \gamma_{5} \kappa_{2}^{d A} / M_{Z}+\left(i \sigma^{\mu \nu} p_{Z \nu}\right) \kappa_{3}^{d} / M_{Z}\right]
\end{aligned}
$$

where

$$
\begin{aligned}
& g_{V}^{d}=-1 / 2+2 / 3 s_{w}^{2} \\
& g_{A}^{d}=-1 / 2
\end{aligned}
$$

and

$$
\begin{aligned}
\kappa_{1}^{d V} & =-4 \frac{M_{Z}^{2}}{e^{2} \Lambda^{2}}\left(f_{\Phi q}^{(1)}+f_{\Phi q}^{(3)}+f_{\Phi d}\right) s_{w}^{2} c_{w}^{2} \\
\kappa_{1}^{d A} & =-4 \frac{M_{Z}^{2}}{e^{2} \Lambda^{2}}\left(f_{\Phi q}^{(1)}+f_{\Phi q}^{(3)}-f_{\Phi d}\right) s_{w}^{2} c_{w}^{2} \\
\kappa_{2}^{d V} & =\frac{M_{Z}^{2}}{e \Lambda^{2}}\left(-f_{D d}+f_{\bar{D} d}\right) s_{w} c_{w} / \sqrt{2} \\
\kappa_{2}^{d A} & =\frac{M_{Z}^{2}}{e \Lambda^{2}}\left(f_{D d}+f_{\bar{D} d}\right) s_{w} c_{w} / \sqrt{2} \\
\kappa_{3}^{d} & =4 \sqrt{2} \frac{M_{Z}^{2}}{e^{2} \Lambda^{2}}\left(f_{d W} c_{w}+f_{d B} s_{w}\right) s_{w}^{2} c_{w}^{2}
\end{aligned}
$$

and

$$
\begin{aligned}
\Gamma_{\mu}^{Z c \bar{c}}= & i \frac{e}{2 s_{W} c_{W}}\left[\gamma^{\mu}\left(g_{V}^{u}+g_{A}^{u} \gamma_{5}\right)-\gamma^{\mu}\left(\kappa_{1}^{u V}-\kappa_{1}^{u A} \gamma_{5}\right) / 2-\left(p_{1}^{\mu}-p_{2}^{\mu}\right) \kappa_{2}^{u V} / M_{Z}-\right. \\
& \left.\left(p_{1}^{\mu}+p_{2}^{\mu}\right) \gamma_{5} \kappa_{2}^{u A} / M_{Z}-\left(i \sigma^{\mu \nu} p_{Z \nu}\right) \kappa_{3}^{u} / M_{Z}\right]
\end{aligned}
$$

where

$$
\begin{aligned}
& g_{V}^{u}=1 / 2-4 / 3 s_{w}^{2} \\
& g_{A}^{u}=1 / 2
\end{aligned}
$$

and 


$$
\begin{aligned}
\kappa_{1}^{u V} & =3 \frac{M_{Z}^{2}}{e^{2} \Lambda^{2}}\left(f_{\Phi q}^{(1)}-f_{\Phi q}^{(3)}+f_{\Phi u}\right) s_{w}^{2} c_{w}^{2} \\
\kappa_{1}^{u A} & =3 \frac{M_{Z}^{2}}{e^{2} \Lambda^{2}}\left(f_{\Phi q}^{(1)}-f_{\Phi q}^{(3)}-f_{\Phi u}\right) s_{w}^{2} c_{w}^{2} \\
\kappa_{2}^{u V} & =\frac{M_{Z}^{2}}{e \Lambda^{2}}\left(-f_{D u}+f_{\bar{D} u}\right) \cos \left(2 \theta_{W}\right) s_{w} c_{w} / \sqrt{2} \\
\kappa_{2}^{u A} & =\frac{M_{Z}^{2}}{e \Lambda^{2}}\left(f_{D u}+f_{\bar{D} u}\right) \cos \left(2 \theta_{W}\right) s_{w} c_{w} / \sqrt{2} \\
\kappa_{3}^{u} & =4 \sqrt{2} \frac{M_{Z}^{2}}{e^{2} \Lambda^{2}}\left(f_{u W} c_{w}-f_{u B} s_{w}\right) s_{w}^{2} c_{w}^{2}
\end{aligned}
$$

The vertex $W^{-} u \bar{d}$ can be written as follows:

$$
\begin{aligned}
\Gamma_{\mu}^{W^{-} u \bar{d}}= & i \frac{e V_{u d}}{2 \sqrt{2} s_{W}}\left[\left(1-\gamma_{5}\right) \gamma_{\mu}+\gamma_{\mu}\left(\kappa_{4}^{u d V}-\kappa_{4}^{u d A} \gamma_{5}\right)-\left(\kappa_{1}^{u d V}-\kappa_{1}^{u d A} \gamma_{5}\right) p_{\bar{d}}^{\mu} / M_{W}\right. \\
& \left.-\left(\kappa_{2}^{u d V}-\kappa_{2}^{u d A} \gamma_{5}\right) p_{u}^{\mu} / M_{W}-\left(\kappa_{3}^{u d V}+\kappa_{3}^{u d A} \gamma_{5}\right) i \sigma^{\mu \nu} p_{W \nu} / M_{W}\right]
\end{aligned}
$$

where

$$
\begin{aligned}
\kappa_{1}^{u d V} & =\frac{\sqrt{2} s_{w} M_{W}^{2}}{e \Lambda^{2}}\left(f_{\bar{d} u}-f_{D d}\right) \\
\kappa_{1}^{u d A} & =\frac{\sqrt{2} s_{w} M_{W}^{2}}{e \Lambda^{2}}\left(f_{\bar{d} u}+f_{D d}\right) \\
\kappa_{2}^{u d V} & =\frac{\sqrt{2} s_{w} M_{W}^{2}}{e \Lambda^{2}}\left(f_{D u}-f_{\bar{d} d}\right) \\
\kappa_{2}^{u d A} & =\frac{\sqrt{2} s_{w} M_{W}^{2}}{e \Lambda^{2}}\left(f_{D u}+f_{\bar{d} d}\right) \\
\kappa_{3}^{u d V} & =\frac{4 \sqrt{2} s_{w} M_{W}^{2}}{e^{2} \Lambda^{2}}\left(f_{u W}+f_{d W}\right) s_{w} \\
\kappa_{3}^{u d A} & =\frac{4 \sqrt{2} s_{w} M_{W}^{2}}{e^{2} \Lambda^{2}}\left(f_{u W}-f_{d W}\right) s_{w} \\
\kappa_{4}^{u d V} & =\frac{2 s_{w}^{2} M_{W}^{2}}{e^{2} \Lambda^{2}}\left(f_{\Phi \Phi}+2 f_{\Phi q}^{3}\right) \\
\kappa_{4}^{u d A} & =\frac{2 s_{w}^{2} M_{W}^{2}}{e^{2} \Lambda^{2}}\left(f_{\Phi \Phi}-2 f_{\Phi q}^{3}\right)
\end{aligned}
$$

The results obtained for the $Z$ decay width into $u$ - and $d$-type and for $W$ decay into quarks are shown in the Appendix, where we include finite quark mass effects and the appropriate QCD and QED radiative corrections.

From these expressions we note some interesting features:

- Only $\kappa_{1}^{u(V, A)}, \kappa_{1}^{d(V, A)}$ and $\kappa_{3}^{u d(V, A)}$ interfere with the Standard Model result. The other 
combinations only appear quadratically and therefore should be less constrained by data. - There could be cancellations in the linear combinations of the coefficients of the effective lagrangian that would the widths less sensitive to them, but we don't consider this possibility further.

- The operator $\mathcal{O}_{\Phi \Phi}$ is not constrained by $Z$ decays.

We get bounds on the different coefficients by requiring $3 \sigma$ deviation criteria for $Z$ and $W$ decay widths. For the $Z$ boson, we consider deviations in the total hadronic width due to anomalous couplings of the first family only and also deviations in $R_{c}$ and $R_{b}$ arising from anomalous couplings of the second and third family respectively. For the $W$ boson we considered deviations in the total width due to anomalous couplings in the first generation. We used the data [16]:

$$
\Gamma(Z)_{\text {hadrons }}=1.7432 \pm 0.0023 \mathrm{GeV} ; \quad R_{b}=0.2170 \pm 0.0009 ; \quad R_{c}=0.1734 \pm 0.0048
$$

and

$$
\Gamma(W)_{\text {total }}=2.07 \pm 0.06 \mathrm{GeV}
$$

The limits obtained are presented in Tables I and II. We clearly see a hierarchy in the constraints: the coefficients $f_{\Phi q}^{(1,3)}, f_{\Phi(u, d)}$ are tightly bounded to be less than $1 \mathrm{TeV}^{-2}$ in most cases, $f_{(u, d)(W, B)}$ are roughly less than a few $\mathrm{TeV}^{-2}$, whereas $f_{\Phi \Phi}, f_{D(u, d)}, f_{\bar{D}(u, d)}$ are typically constrained to be less than $20 \mathrm{TeV}^{-2}$.

We would like to stress that the limits shown below (especially for the third quark's generation) exclude several operators for anomalous top-quark and Higgs physics with precision which is out of range for the present and future hadron colliders. For example, limits on the couplings $f_{\Phi q}^{(1,3)}, f_{D d}, f_{\bar{D} d}$ and $f_{d W}$ bound different structures of $W t b$ anomalous coupling, as also noted recently in [13] (however, apparently they did not consider all possible structures in the interaction vertices). All couplings we considered are also related to anomalous Higgs interactions with quarks and gauge bosons like $H q q, H V q q$. Therefore the operators considered in this paper should be taken into account if one study anomalous Higgs couplings effects at the colliders. 


\section{CONCLUSIONS}

The search for the effect of higher dimensional operators that give rise to anomalous couplings should be pursued in all possible processes since the results may provide important information on physics beyond the Standard Model. However, one should not forget that the contribution of some of these operators are strongly bounded due to precision measurements at LEP.

In this note we derived limits on the coefficients of a complete set of dimension- 6 operators involving electroweak gauge bosons, quarks and Higgs fields. We showed the linear combinations of these operators that are important for the decay processes. In particular, there are some operators that are not tightly bound by the gauge boson decay widths but they could be important for other processes like anomalous associated Higgs production at the Tevatron.

\section{ACKNOWLEDGMENTS}

One of the authors (A.S.B.) is grateful to A. V. Semenov for important improvements of the LanHEP package. This work was supported by Conselho Nacional de Desenvolvimento Científico e Tecnológico (CNPq), and by Fundação de Amparo à Pesquisa do Estado de São Paulo (FAPESP). 


\section{REFERENCES}

[1] S. Weinberg, Physica 96A, 327 (1979); se also H. Georgi, Weak Interactions and Modern Particle Theory (Benjamim/Cummings, Menlo Park, 1984) and J. F. Donoghue, E. Golowich and B. R. Holstein, Dynamics of the Standard Model (Cambridge University Press, 1992).

[2] W. Buchmüller and D. Wyler, Nucl. Phys. B268, 621 (1986); C. J. C. Burgess and H. J. Schnitzer, Nucl. Phys. B228, 454 (1983); C. N. Leung, S. T. Love and S. Rao, Z. Phys. 31, 433 (1986); A. De Rújula, M. B. Gavela, P. Hernández and E. Massó, Nucl. Phys. B384, 3 (1992), C. Arzt, M. B. Einhorn and J. Wudka, Nucl. Phys. B433, 41 (1995) .

[3] K. Hagiwara, S. Ishihara, R. Szalapski and D. Zeppenfeld, Phys. Lett. B283, 353 (1992); idem, Phys. Rev. D48, 2182 (1993).

[4] K. Hagiwara, R. Szalapski and D. Zeppenfeld, Phys. Lett. B318, 155 (1993).

[5] K. Hagiwara and M. L.Stong, Z. Phys. 62, 99 (1994); G. J. Gounaris, F. M. Renard and N. D. Vlachos, Nucl. Phys. B459, 51 (1996).

[6] S. M. Lietti, S. F. Novaes and R. Rosenfeld, Phys. Rev. D54, 3266 (1996); F. de Campos, S. M. Lietti, S. F. Novaes and R. Rosenfeld, Phys. Lett. B389, 93 (1996).

[7] G. J. Gounaris, J. Layssac and F. M. Renard, Z. Phys. 69, 505 (1996); G. J. Gounaris and F. M. Renard, Z. Phys. 69, 513 (1996).

[8] G. A. Leil and W. J. Stirling, J. Phys. G21, 517 (1995).

[9] W. Killian, M. Krämer and P. M. Zerwas, Phys. Lett. B381, 243 (1996); B. Grzadowski and J. Wudka, Phys. Lett. B364, 49 (1995).

[10] F. de Campos, M.C. Gonzalez-Garcia, S.F. Novaes, Phys.Rev.Lett.79, 5210 (1997)

[11] M.C. Gonzalez-Garcia, A. Gusso, S.F. Novaes,IFT-P-011-98, hep-ph/9802254 
[12] K. Wishnant, J. M. Yang, B. -L. Young and X. Zhang, Phys. Rev. D56, 467 (1997)

[13] J. -J. Cao, J. -X. Wang, J. M. Yang, B. -L. Young and X. Zhang, hep-ph/9804343.

[14] E. E. Boos, M. N. Dubinim, V. A. Ilyin, A. E. Pukhov and V. I. Savrin, preprint INP MSU 94-36/358, hep-ph/9503280.

[15] A. V. Semenov, preprint INP MSU 96-24/431.

[16] The LEP Electroweak Working Group, CERN-PPE/97-154 (1997). 


\section{TABLES}

\begin{tabular}{||l||r|r|r||}
\hline \hline \multirow{1}{|||}{ Anomalous Couplings } & \multicolumn{3}{c||}{ Limits } \\
\hline & 1-st generation & 2-nd generation & 3-rd generation \\
\hline \hline$f_{\Phi q}^{(1)} / \Lambda^{2}$ & $(-0.17,0.17)$ & $(-0.56,0.58)$ & $(-0.092,0.092)$ \\
\hline$f_{\Phi q}^{(3)} / \Lambda^{2}$ & $(-0.10,0.10)$ & $(-0.59,0.56)$ & $(-0.093,0.092)$ \\
\hline$f_{\Phi u} / \Lambda^{2}$ & $(-0.54,0.54)$ & $(-1.6,1.2)$ & - \\
\hline$f_{\Phi d} / \Lambda^{2}$ & $(-0.65,0.90)$ & - & $(-0.50,0.63)$ \\
\hline$f_{\Phi \Phi} / \Lambda^{2}$ & $(-26,26)$ & $(-52,49)$ & - \\
\hline$f_{D u} / \Lambda^{2}$ & $(-14,14)$ & - & $(-17,8.8)$ \\
\hline$f_{D d} / \Lambda^{2}$ & $(-26,26)$ & $(-49,52)$ & - \\
\hline$f_{\bar{D} u} / \Lambda^{2}$ & $(-14,14)$ & - & $(-8.8,17)$ \\
\hline$f_{\bar{D} d} / \Lambda^{2}$ & $(-1.6,1.6)$ & $(-3.2,2.7)$ & - \\
\hline$f_{u W} / \Lambda^{2}$ & $(-2.9,2.9)$ & $(-5.1,6.0)$ & - \\
\hline$f_{u B} / \Lambda^{2}$ & $(-1.6,1.6)$ & - & $(-3.2,0.53)$ \\
\hline$f_{d W} / \Lambda^{2}$ & $(-2.9,2.9)$ & - & - \\
\hline$f_{d B} / \Lambda^{2}$ & - & - \\
\hline \hline
\end{tabular}

TABLE I. Intervals of allowed values of the coefficients $f_{i} / \Lambda^{2}$ in units of $\mathrm{TeV}^{-2}$ using $3 \sigma$ deviation criteria for $\Gamma(Z \rightarrow$ hadrons $)(\Gamma(Z \rightarrow \bar{c} c))$ for coefficients of the 1-st and second quark's generation and for $\Gamma(Z \rightarrow \bar{b} b)$ for coefficients of the 3-rd quark's generation. 


\begin{tabular}{||l||r||}
\hline \hline Anomalous Couplings & \multicolumn{1}{c||}{ Limits } \\
\hline \hline$f_{\Phi \Phi} / \Lambda^{2}$ & $(-17,17)$ \\
\hline$f_{D u} / \Lambda^{2}$ & $(-104,104)$ \\
\hline$f_{D d} / \Lambda^{2}$ & $(-104,104)$ \\
\hline$f_{\bar{D} u} / \Lambda^{2}$ & $(-104,104)$ \\
\hline$f_{\bar{D} d} / \Lambda^{2}$ & $(-104,104)$ \\
\hline$f_{u W} / \Lambda^{2}$ & $(-8.7,8.7)$ \\
\hline$f_{d W} / \Lambda^{2}$ & $(-8.7,8.7)$ \\
\hline \hline
\end{tabular}

TABLE II. Intervals of allowed values of the coefficients $f_{i} / \Lambda^{2}$ in units of $\mathrm{TeV}^{-2}$ using $3 \sigma$ deviation criteria for $\Gamma(W)_{\text {total }}$. 


\section{APPENDIX}

In this appendix we collect the results for the widths, including finite mass effects and radiative corrections.

The $Z$ partial decay width into $u$-type quarks is given by:

$$
\begin{aligned}
\Gamma(Z \rightarrow U \bar{U})= & \frac{e^{2} M_{Z}}{64 \pi c_{w}^{2} s_{w}^{2}} \sqrt{\left(1-4 M_{U}^{2} / M_{Z}^{2}\right)}\left[4\left(\left(g_{A}^{u}\right)^{2}+\left(g_{V}^{u}\right)^{2}\right)-4\left(g_{V}^{u} \kappa_{1}^{u V}-g_{A}^{u} \kappa_{1}^{u A}\right)+\right. \\
& \left.\left(\kappa_{1}^{u A}\right)^{2}+\left(\kappa_{1}^{u V}\right)^{2}+2\left(\kappa_{2}^{u V}\right)^{2}-4 \kappa_{2}^{u V} \kappa_{3}^{u}+2\left(\kappa_{3}^{u}\right)^{2}+\Delta_{U}\right]
\end{aligned}
$$

Finite quark mass effects are described by the function:

$$
\begin{aligned}
\Delta_{U}= & {\left[32\left(\kappa_{2}^{u V}\right)^{2} M_{U}^{4} / M_{Z}^{4}+16\left(2 g_{V}^{u}-\kappa_{1}^{u V}\right) \kappa_{2}^{u V} M_{U}^{3} / M_{Z}^{3}+\right.} \\
& 2\left(4\left(g_{V}^{u}\right)^{2}-8\left(g_{A}^{u}\right)^{2}+4\left(2 g_{A}^{u} \kappa_{1}^{u A}-g_{V}^{u} \kappa_{1}^{u V}\right)-2\left(\kappa_{1}^{u A}\right)^{2}+\left(\kappa_{1}^{u V}\right)^{2}-\right. \\
& \left.\left.8\left(\left(\kappa_{2}^{u V}\right)^{2}-\kappa_{2}^{u V} \kappa_{3}^{u}-\left(\kappa_{3}^{u}\right)^{2}\right)\right) M_{U}^{2} / M_{Z}^{2}+4\left(-2 g_{V}^{u}+\kappa_{1}^{u V}\right)\left(\kappa_{2}^{u V}-3 \kappa_{3}^{u}\right) M_{U} / M_{Z}\right]
\end{aligned}
$$

The $Z$ partial decay width into $d$-type quarks is given by:

$$
\begin{aligned}
\Gamma(Z \rightarrow D \bar{D})= & \frac{e^{2} M_{Z}}{64 \pi c_{w}^{2} s_{w}^{2}} \sqrt{\left(1-4 M_{D}^{2} / M_{Z}^{2}\right)}\left[4\left(\left(g_{A}^{d}\right)^{2}+\left(g_{V}^{d}\right)^{2}\right)+4\left(g_{V}^{d} \kappa_{1} d V+g_{A}^{d} \kappa_{1}^{d A}\right)+\right. \\
& \left.\left(\kappa_{1}^{d A}\right)^{2}+\left(\kappa_{1}^{d V}\right)^{2}+2\left(\kappa_{2}^{d V}\right)^{2}-4 \kappa_{2}^{d V} \kappa_{3}^{d}+2\left(\kappa_{3}^{d}\right)^{2}+\Delta_{D}\right]
\end{aligned}
$$

Finite quark mass effects are described by the function:

$$
\begin{aligned}
\Delta_{D}= & {\left[32\left(\kappa_{2}^{d V}\right)^{2} M_{D}^{4} / M_{Z}^{4}-16\left(2 g_{V}^{d}+\kappa_{1}^{d V}\right) \kappa_{2}^{d V} M_{D}^{3} / M_{Z}^{3}+\right.} \\
& 2\left(4\left(g_{V}^{d}\right)^{2}-8\left(g_{A}^{d}\right)^{2}+4\left(-2 g_{A}^{d} \kappa_{1}^{d A}+g_{V}^{d} \kappa_{1}^{d V}\right)-2\left(\kappa_{1}^{d A}\right)^{2}+\left(\kappa_{1}^{d V}\right)^{2}-\right. \\
& \left.\left.8\left(\left(\kappa_{2}^{d V}\right)^{2}-\kappa_{2}^{d V} \kappa_{3}^{d}-\left(\kappa_{3}^{d}\right)^{2}\right)\right) M_{D}^{2} / M_{Z}^{2}+4\left(2 g_{V}^{d}+\kappa_{1}^{d V}\right)\left(\kappa_{2}^{d V}-3 \kappa_{3}^{d}\right) M_{D} / M_{Z}\right]
\end{aligned}
$$

The $W$-boson partial decay width into $\bar{d}$ and $u$ type quarks is given by: and for W decay:

$$
\begin{aligned}
\Gamma\left(W^{+} \rightarrow u \bar{d}\right)= & \frac{e^{2} V_{u d}^{2}}{256 \pi} \frac{M_{W}}{s_{w}^{2}}\left[8\left(1+\kappa_{4}^{u d V}\right)^{2}+8\left(1+\kappa_{4}^{u d A}\right)^{2}+\right. \\
& \left.\left(-\kappa_{1}^{u d V}+\kappa_{2}^{u d V}+2 \kappa_{3}^{u d V}\right)^{2}+\left(-\kappa_{1}^{u d A}+\kappa_{2}^{u d A}+2 \kappa_{3}^{u d A}\right)^{2}\right] .
\end{aligned}
$$

We incorporate the appropriate QCD and QED radiative corrections by including the factors: 


$$
\begin{aligned}
\delta_{Q C D} & =1+\frac{\alpha_{s}}{\pi}+1.409 \frac{\alpha_{s}^{2}}{\pi^{2}}-12.77 \frac{\alpha_{s}^{3}}{\pi^{3}}=1.03954 \\
\delta_{Q E D} & =1+\frac{3 \alpha Q_{f}^{2}}{4 \pi} \\
\delta_{b \bar{b}} & =1+0.01\left(-\frac{m_{t}^{2}}{2 M_{Z}^{2}}+\frac{1}{5}\right)=0.98359
\end{aligned}
$$

For our numerical results showed in Tables I and II we used $\alpha_{s}=0.120, \alpha=\frac{e^{2}}{4 \pi}=1 / 128$, $m_{t}=175 \mathrm{GeV}, m_{b}=4.3 \mathrm{GeV}, m_{c}=1.3 \mathrm{GeV}, s_{w}=0.473$ and $M_{Z}=91.1867 \mathrm{GeV}$. 